\title{
Transtorno de humor bipolar: diversas apresentações de uma mesma doença
}

\author{
Bipolar mood disorder: different occurrences of the same disease
}

\author{
Audrey Regina Magalhães Braga ${ }^{1}$, Lia Silvia Kunzler ${ }^{2}$, Feng Yu Hua ${ }^{3}$ \\ ${ }^{1}$ Especialista em Saúde e Educação. Médica, Secretaria Estadual de Saúde, Brasília, DF. ${ }^{2}$ Especialista em Psiquiatria, Associação Brasileira de \\ Psiquiatria e Associação Médica Brasileira. Terapeuta cognitiva, Beck Institute, Filadélfia, EUA. Psiquiatra, Serviço de Assistência Médica, \\ Decanato de Assuntos Comunitários, Universidade de Brasília (UnB), Brasília, DF. ${ }^{3}$ Especialista em Neuropsicologia. Neuropsicóloga, Mental Help \\ - Clínica de Psiquiatria e Psicologia, Brasília, DF.
}

\begin{abstract}
Resumo
O presente relato tem por objetivo acompanhar a evolução de um caso que se apresentou como mutismo seletivo e evoluiu para transtorno de humor bipolar e chamar atenção para a diversidade de sintomas possíveis no transtorno de humor. Em geral, o mutismo seletivo tem início na idade pré-escolar, porém os sintomas chamam mais atenção na idade escolar. A importância do diagnóstico e tratamento precoces reside na prevenção das complicações, tais como distúrbios no desenvolvimento social e acadêmico e na autoestima, além da possibilidade de evolução para outros transtornos de ansiedade. O transtorno de humor bipolar é uma doença mental caracterizada por variações extremas no humor. Na criança, prejudica o crescimento emocional e seu desenvolvimento. É confundido com transtorno de déficit de atenção/hiperatividade e transtornos de comportamento, tais como transtorno de conduta e desafiador opositivo. A paciente do sexo feminino, quando avaliada, estava com 5 anos e 9 meses de idade. Concluiu-se o diagnóstico como mutismo seletivo. Foi indicada psicoterapia, com orientações aos pais e à escola, e iniciado o uso de inibidores seletivos de recaptação da serotonina, com boa resposta aos sintomas de ansiedade, mas passou a apresentar piora significativa do comportamento. Foi levantada a hipótese diagnóstica de transtorno de humor bipolar de início precoce. Medicada com estabilizador, apresentou adequação na terceira medicação e evolução satisfatória. O mutismo seletivo, considerado um transtorno de ansiedade na infância, pode ser pródromo para outros quadros psiquiátricos na infância. Há necessidade que os pediatras, médicos que primeiro acessam essas crianças, assim como os psiquiatras da infância, estejam atentos à riqueza de sintomas que pode dar seguimento ao quadro.
\end{abstract}

Descritores: Criança, mutismo, transtorno bipolar, terapia cognitiva.

\begin{abstract}
The purpose of the present case report is to follow the evolution of a child who presented selective mutism and evolved into bipolar mood disorder, and also to draw attention to the diversity of possible symptoms of mood disorders. In general, selective mutism starts at preschool age, although the symptoms are more evident at school age. The importance of an early diagnosis and treatment lies in the prevention of some complications, such as social and academic development and self-esteem, besides the possibility of the development of other anxiety disorders. Bipolar mood disorder is a mental disease characterized by extreme mood variations. In childhood, it hinders the child's emotional growth and development. Selective mutism is mistaken by attention deficit hyperactivity disorder, and behavioral disorders, such as conduct disorder and oppositional defiance disorder. The present case report is about a female patient evaluated at 5 years and 9 months of age. We concluded the case as being a selective mutism disorder. Not only psychotherapy, with parent and school orientation, but also medication was prescribed. The first prescribed medication was use of selective serotonin reuptake inhibitors with good results regarding anxiety symptoms, although the patient started to present significant deterioration in behavior. Because of that, the diagnostic hypothesis came to be bipolar mood disorder with an early start. The patient presented satisfactory evolution only after given a third option of mood stabilizer medication. Selective mutism, which is an anxiety disorder in childhood, can be prodromus to other psychiatric conditions at the same age. It is necessary for pediatricians, who are the doctors that first assess these children, and also child psychiatrists, to be aware of the wealth of symptoms that can give continuation to the condition.
\end{abstract}

Keywords: Child, mutism, bipolar disorder, cognitive therapy.

Audrey Regina Magalhães Braga, Centro Empresarial Brasília, SRTVS, 701, bloco C, sala 204, Asa Sul, CEP 70340-907, Brasília, DF. E-mail: audrey64@globo.com 


\section{Introdução}

O transtorno de humor bipolar (THB) é descrito desde meados do século XIX, porém na última década recebeu maior atenção da comunidade científica, sendo reconhecido como patologia presente na população mais jovem ${ }^{1}$.

A apresentação clínica do THB na infância e adolescência é diferente da manifestação na vida adulta. Há controvérsias quanto aos sintomas característicos do quadro naquela população ${ }^{2,3}$, o que contribui para a divergência da existência do mesmo ${ }^{1}$. O THB pré-puberal pode se apresentar da seguinte forma: humor irritável ${ }^{4}$ com tempestades afetivas, curso crônico ${ }^{5}$, sintomas mistos de depressão e mania ${ }^{2}$, ciclagem rápida, baixa recuperação interepisódica ${ }^{6}$, labilidade emocional e irritabilidade durante todos os episódios ${ }^{7-9}$. A criança é ativa e impulsiva, explosiva e irritada, fala em excesso, muda rapidamente de assunto, tem pouca necessidade de sono. Acredita ter habilidades especiais e/ou poder fazer coisas irreais ${ }^{10,11}$. As crianças em idade escolar e os adolescentes em geral apresentam humor irritável, mania mista, ciclagem rápida, delírios de grandeza, labilidade emocional e explosões de raiva. Na literatura atual, os dados revelam igual prevalência de fenômenos psicóticos na criança e no adolescente ${ }^{12}$, com altas taxas de suicídio ${ }^{13}$, repetência escolar, agressão, comportamentos de alto risco, como promiscuidade sexual e abuso de substâncias, e altas taxas de recorrência ${ }^{6}$.

A complexidade e a variedade dos sintomas atrasam e confundem o diagnóstico, resultando no atraso do tratamento específico e adequado $0^{5,6,14}$.

O THB freqüentemente se sobrepõe ou ocorre juntamente com outras desordens: transtorno de déficit de atenção/hiperatividade (TDAH) em 80 a $90 \%$ das crianças; e em $30 \%$ dos adolescentes ${ }^{1,15}$, depressão com sintomas muito intensos, elevado risco de suicídio ${ }^{1}$ e ansiedade ${ }^{4,6,16-19}$.

O diagnóstico incorreto expõe o paciente ao uso de psicoestimulantes e/ou antidepressivos, e o tardio pode torná-lo resistente ao tratamento, que, quando ocorre de forma adequada, objetiva a inclusão social e acadêmica do paciente ${ }^{20}$.

O mutismo seletivo (MS) foi descrito em 1877 por Kussmaul como afasia voluntária em pessoas que não falavam em algumas situações, apesar de possuírem a habilidade de fazê-lo. Acreditava-se, inicialmente, que as pessoas optavam por não falar em determinadas circunstâncias. Em 1934, Tramer utilizou o termo mutismo eletivo para descrever crianças que só se comunicavam em determinadas situações ou com certas pessoas, mas não falavam na maior parte das situações sociais. Sugeriu-se que era uma recusa em falar, caracterizando um comportamento de oposição ${ }^{21}$. Com a maior compreensão do quadro, o MS passou a ser reconhecido como uma desordem de ansiedade na infância ${ }^{22}$ que apresenta como característica principal o fracasso persistente em falar em situações sociais específicas, ambientes pouco familiares ${ }^{21}$, na escola e com amigos, onde seria esperado que falasse, apesar de falar em outras situações ${ }^{23-25}$.

É freqüente a comorbidade com fobia social (FS), caracterizada por intensa ansiedade gerada por situações que geram comportamentos evitativos frente às mesmas $^{21}$. Apesar da recusa em falar, a criança com MS - termo adotado com a publicação do Manual de Diagnóstico e Estatística das Perturbações Mentais (DSM-IV) - apresenta interesse na comunicação, tentando estabelecê-la através de gestos, desenhos, acenos e balanços de cabeça. Os subtipos incluem: tímido, fóbico ou ansioso, hostil ou agressivo, além de um subtipo reativo, histérico ou traumático ${ }^{26}$.

O funcionamento social e acadêmico dessas crianças pode ficar prejudicado se não tratado adequadamente, mesmo que haja alguma melhora com o tempo ${ }^{27,28}$.

Tem sido associado com várias outras patologias psiquiátricas da infância, alterações no desenvolvimento, especialmente da linguagem, encoprese, enurese e transtorno de integração sensorial, diagnóstico encontrado em crianças autistas ${ }^{21,26}$. Os primeiros sintomas se manifestam entre os 3 e 8 anos, embora freqüentemente a criança não chegue à atenção clínica antes do período escolar ${ }^{26}$. É mais freqüente em meninas, numa proporção de $2: 1^{21}$.

$\mathrm{O}$ diagnóstico precoce permite as intervenções terapêuticas com o objetivo de diminuir os sintomas, melhorar a qualidade de vida e prevenir os transtornos ansiosos na vida adulta ${ }^{21}$. Modificação comportamental e psicofarmacoterapia com inibidores seletivos de recaptação da serotonina (ISRS) são opções de tratamento ${ }^{28}$.

$\mathrm{O}$ presente relato descreve um caso de MS como parte da diversidade de apresentação do THB de início na infância.

\section{Relato do caso}

Paciente do sexo feminino, encaminhada para avaliação aos 5 anos e 9 meses, com queixa de ausência de comunicação verbal quando fora do ambiente familiar. Pais negavam qualquer dificuldade na linguagem receptiva e/ou expressiva. É primogênita de uma prole de dois filhos, sendo o segundo do sexo masculino, sem alterações de saúde física e/ou mental. A criança foi descrita como muito inteligente, de temperamento forte e comportamento opositor que não preenchia critérios diagnósticos para transtorno desafiador opositivo.

A coleta de dados deve constar das informações dos pais e/ou cuidadores, da criança e, quando necessário, de professores e outros profissionais que atendam a criança ${ }^{29}$. As informações acadêmicas 
poderão ser úteis para avaliação de progresso de tratamento e funcionamento geral ${ }^{29}$.

Neste caso, a avaliação médica consistiu de dois encontros com os pais, quatro sessões com a criança, discussão de caso com a profissional que a encaminhou, avaliação da escola e observação da criança em situações sociais: recreio escolar e sua festa de formatura. A conclusão diagnóstica foi MS.

Indicou-se acompanhamento semanal, que consistiu em sessões de 60 minutos cada, baseadas na terapia cognitivo-comportamental (TCC). Essa técnica apresenta evidências favoráveis para o tratamento de vários transtornos, incluindo os de ansiedade ${ }^{30-32}$. Nos primeiros 3 meses de acompanhamento, a paciente não apresentou comunicação oral, usando a mãe como intermediadora, com sussurros. Seu contato visual era tímido, mas consistente. Passou a utilizar a comunicação escrita, e a seguir vieram os sussurros dirigidos à profissional, com progressivo aumento do tom de voz. O principal foco de aproximação com a paciente foi o interesse comum pelos cavalos, utilizado como brincadeiras, e foi descoberto pela paciente como prática desportiva - equitação. $\mathrm{Na}$ escola, permaneceu sem prejuízo no funcionamento acadêmico.

Durante o acompanhamento, os pais referiram intenso sofrimento emocional frente às situações de exposição social, que era percebido por palidez cutânea, sudorese, resistência a participar de algumas situações sociais e queixa de taquicardia. Em função do descrito e do diagnóstico, foi indicada medicação antidepressiva - ISRS ${ }^{28,30}$. Após avaliação clínica e laboratorial da paciente, cujo objetivo foi afastar possíveis condições médicas e estabelecer perfil metabólico basal para exames de controle periódicos no futuro $^{29} \mathrm{com}$ resultados normais, iniciou-se paroxetina 10 mg pela manhã até a dose máxima de $20 \mathrm{mg} / \mathrm{dia}$ - sem efeitos colaterais. A paciente apresentou boa resposta à mesma, com comportamentos sociais mais adequados.

Após 6 meses de uso da medicação, os pais referiram piora significativa do comportamento opositor, labilidade de humor, com explosões de raiva que podiam ser longas e por motivos banais, agressividade verbal e eventualmente física, distratibilidade e atenção instável, mais energia, mais autocentrada, além de grandiosidade e egocentrismo em algumas situações. Durante as sessões, esses sintomas também foram observados, e chamou atenção o maior interesse por assuntos de $\operatorname{sexo}^{33}$, meninos e namoro, não compatível com sua idade e comportamento anterior. Quando questionados, os pais referiram a mesma preocupação e confirmaram a ausência desse comportamento em outros momentos. Não houve evidência que sugerisse abuso sexual.

Durante o tratamento, o pai foi avaliado e diagnosticado como portador de TDAH e a mãe como tendo depressão recorrente e resistente, e ambos iniciaram tratamento medicamentoso. Acrescentaram- se os seguintes dados na história familiar: avô materno descrito como impulsivo e agressivo verbalmente, sem tratamento psiquiátrico; avô paterno alcoolista e abusivo, também sem tratamento.

Levantou-se a hipótese diagnóstica de THB, de início precoce, mais claramente definido após o uso de antidepressivo, compatível com a literatura atual ${ }^{5,7,34}$.

Indicou-se estabilizador de humor, sendo opção a oxcarbazepina, que até a dosagem de $900 \mathrm{mg} / \mathrm{dia}$ não apresentou a resposta esperada. $\mathrm{O}$ segundo, divalproato de sódio, na dosagem de $500 \mathrm{mg} /$ dia, desencadeou importante queda de cabelos, sendo feita a substituição por carbamazepina, que se mostrou adequada a partir de $600 \mathrm{mg} / \mathrm{dia}$.

Uma das intervenções realizadas com a paciente foi a identificação de seus estados de humor ${ }^{29,34}$. A representação dos mesmos está apresentada nas figuras que seguem, feitas em sessão de terapia, e sistematizam o modelo cognitivo dos sintomas depressivos e hipomaníacos (Figura 1).

$\mathrm{Na}$ TCC, a psicoeducação e a conceituação cognitiva são fundamentais. No presente relato, essas técnicas foram trabalhadas utilizando-se o desenho como instrumento para compreensão das distorções cognitivas. Na Figura 1, a paciente desenhou como ela
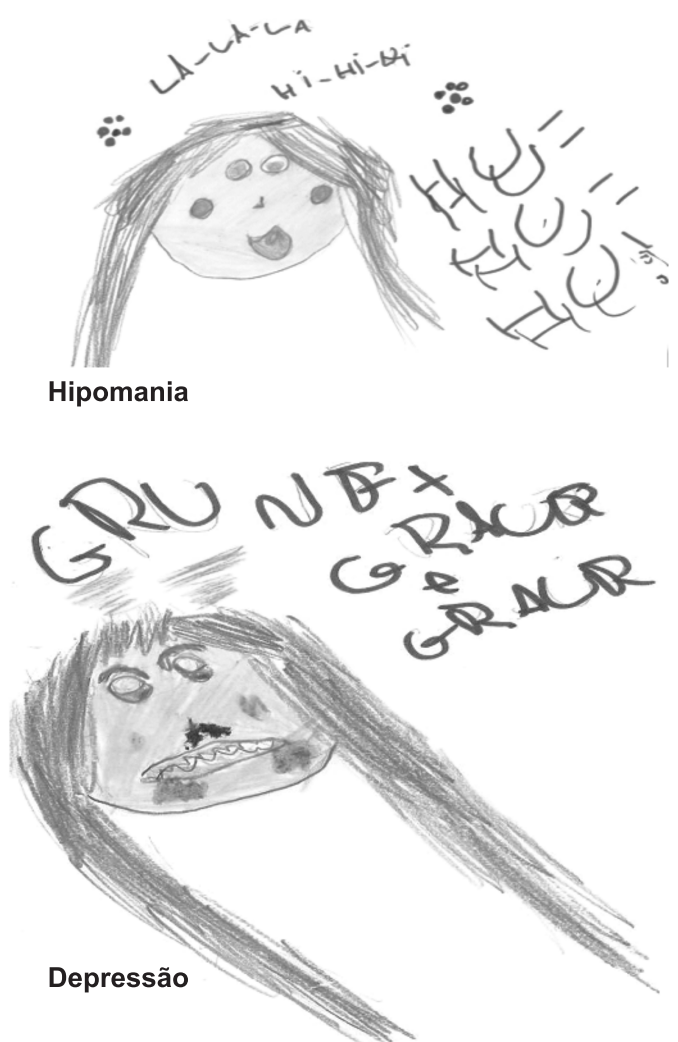

Figura 1 - Sistematização do modelo cognitivo (terapia cognitivo-comportamental) dos sintomas de oscilação do humor 
se conceitua quando em hipomania e em depressão. A conceituação cognitiva, em forma de desenho, permite que os problemas enfrentados e que ocasionaram o encaminhamento para tratamento sejam identificados e que os objetivos específicos sejam determinados.

\section{Discussão}

O MS, sendo considerado um transtorno de ansiedade na infância, pode constituir um pródromo para outros transtornos psiquiátricos. É preciso que os médicos, tanto pediatras quanto psiquiatras, estejam atentos para a sintomatologia, mantendo um adequado seguimento e acompanhamento da criança para tentar assegurar um desenvolvimento mental saudável e garantir a melhor escolha farmacológica. É importante ressaltar que crianças podem ser especialmente sensíveis ao desenvolvimento de mania induzida por ISRS, mais comum em quadros mistos associados a maior risco de suicídio ${ }^{35}$.

A paciente desenvolveu melhora funcional importante, observável na qualidade da adequação social, dos relacionamentos e da vida familiar. Há indicação de manutenção do tratamento, pois, na literatura, há relato de que a associação entre os quadros de ansiedade e humor bipolar na infância predizem um quadro mais grave deste último ${ }^{36}$.

\section{Referências}

1. Boarati MA, Cavalcanti AR, Fu-I L. Uso de quetiapina em transtorno de humor bipolar de início precoce. Rev Psiquiatr RS. 2006;28(3):346-51.

2. Rohde LA, Tramontina S. Tratamento farmacológico do transtorno bipolar na infância e adolescência. Revista de Psiq Clin. 2005;32(Supl 1):117-27.

3. Dickstein DP, Leibenluft E. Emotion regulation in children and adolescents: boundaries between normalcy and bipolar disorder. Dev Psychopathol. 2006;18(4):1105-31.

4. Biederman J, Faraone SV, Wozniak J, Mick E, Kwon A, Cayton GA, et al. Clinical correlates of bipolar disorder in a large referred sample of children and adolescents. J Psychiatr Res. 2005; 39(6):611-22.

5. Papolos DF, Papolos J. The bipolar child. Nova York: Broadway Books; 1999.

6. Petresco S, Ribeiro CR, Pianca TG, Bergman D, Tramontina S, Zavaschi ML, et al. Transtorno bipolar na infância e diagnóstico diferencial con TDAH. Casos Clin Psiiquiatria. 2002;4(1,2):54-60.

7. Akiskal H. Revisão geral do transtorno bipolar. Latinamerican Bipolar Disease Experts Meeting; setembro de 2004; Panamá.

8. Mohr WK. Bipolar disorder in children. J Psychosoc Nurs Ment Health Serv. 2001;39(3):12-23.

9. Geller B, Fox LW, Clark KA. Rate and Predictors of prepubertal bipolarity during follow-up of 6 to 12 year old depressed children. J Am Acad Child Adolesc Psychiatry. 1994;33: 461-8.

10. Barbirato F, Dias G. Transtorno de humor bipolar na infância e na adolescência. Segmentofarma. 2006. (Material de distribuição exclusiva à classe médica; código da publicação 2906.0806.)

11. Carlson GA, Meyer SE. Phenomenology and diagnosis in bipolar disorder in children, adolescents and adults: complexities and developmental issues. Dev Psychopathol. 2006;18 (4): 939-69.
12. Tillman R, Geller B, Klages T, Corrigan M, Bolhofner K, Zimerman B. Psychotic phenomena in 257 young children and adolescents with bipolar I disorder: delusions and hallucinations (benign and pathological). Bipolar Disord. 2008;10(1):45-55.

13. Grunebaum MF, Ramsay SR, Galfalvy HC, Ellis SP, Burke AK, Sher L, et al. Correlates of suicide attempt history in bipolar disorder: a stress-diathesis perspective. Bipolar Disord. 2006;8(5 Pt 2):551-7.

14. Berk M, Berk L, Moss K, Dodd S, Malhi GS. Diagnosing bipolar disorder: how can we do it better? Med J Aust 1. 2006;184(9):459-62.

15. Dickstein DP, Garvey M, Pradella AG, Greenstein DK, Sharp WS, Castellanos FX, et al. Neurologic examination abnormalities in children with bipolar disorder or attention-deficit/ hyperactivity disorder. Biol Psychiatry. 2005;58(7):517-24.

16. Masi G, Toni C, Perugi G, Mucci M, Millepiedi S, Akiskal HS. Anxiety disorders in children and adolescents with bipolar disorder: a neglected comorbidity. Can J Psychiatry. 2001;46(9):797-802.

17. Kaye NS. Is your depressed patient bipolar? J Am Board Fam Pract. 2005;18(4):271-81.

18. Post RM. The impact of bipolar depression. J Clin Psychiatry. 2005;66 Supl 5:5-10

19. Kim EY, Miklowitz DJ. Childhood mania, attention deficit hyperactivity disorder and conduct disorder: a critical review of diagnostic dilemmas. Bipolar Disorder. 2002;4(4):215-25.

20. Chang K, Steiner H, Dienes K, Adleman N, Ketter T. Bipolar offspring: a window into bipolar disorder evolution. Biol Psychiatry. 2003;53(11):945-51.

21. Asbahr FR, organizadores. Transtorno de ansiedade na infância e adolescência. Rio de Janeiro: Elsevier; 2007.

22. Schneier FR, Blanco C, Antia SX, Liebowitz MR. The social anxiety spectrum. Psychiatr Clin North Am. 2002;25 (4):757-74.

23. American Psychiatric Association. Diagnostic and statistical manual of mental disorders. 4th ed. Washington: American Psychiatric Association; 1994.

24. Cohan SL, Price JM, Stein MB. Suffering in silence: why a developmental psychopathology perspective on selective mutism is needed. J Dev Behav Pediatr. 2006;27(4) 341-55.

25. Cohan SL, Chavira DA, Stein MB. Practitioner review: psychosocial interventions for children with selective mutism: a critical evaluation of the literature from 1990-2005. J Chid Psychol Psychiatry. 2006;47(11): 1085-97.

26. Lewis M. Tratado de psiquiatria da infância e adolescência. Porto Alegre: ArtMed; 1995.

27. Bergman RL, Piacentini J, McCracken JT. Prevalence and description of selective mutism in a school-based sample. J Am Acad Child Adolesc Psychiatry. 2002;41(8):938-46.

28. Shwartz RH, Freedy AS, Sheridan MJ. Selective mutism: are primary care physicians missing the silence? Clin Pediatr (Phila). 2006;45(1):43-8.

29. Fu-I L. Transtorno bipolar na infância e na adolescência. São Paulo: Segmento Farma; 2007.

30. Schwartz RH, Shipon-Blum E. "Shy" child? Don't overlook selective mutism [database on the Internet]. Contemp Pediatr. 2005. Disponível em: http://www.selectivemutismcenter.org/ contemp.htm

31. Pheula GF; Isolan, LR. Psicoterapia baseada em evidências em crianças e adolescentes. Rev Psiquiatr Clin. 2007;34(2):74-83.

32. Isolan L, Pheula G, Manfro GG. Tratamento do transtorno de ansiedade social em crianças e adolescentes. Rev. Psiq. Clin. 2007;34(3):125-32.

33. Geller B, Warner K, Williams M, Zimerman B. Prepubertal and young adolescent bipolarity versus ADHD: assessment and validity using the WASH-U-KSADS, CBCL and TRF. J Affect Disord. 1998;51(2):93-100.

34. Geller B, DelBello MP. Bipolar disorder in childhood and early adolescence.. New York: : Guilford; 2003.

35. El-Mallakh RS, Ghaemi SN. Depressão bipolar: um guia abrangente. Porto Alegre: ArtMed; 2008.

36. Dickstein DP, Rich BA, Binstock AB, Pradella AG, Towbin KE, Pine DS, et al. Comorbid anxiety in phenotypes of pediatric bipolar disorder. J Child Adolesc Psychopharmacol. 2005;15(4):534-48. 